\title{
FINITARILY LINEAR WREATH PRODUCTS
}

\author{
B. A. F. WEHRFRITZ \\ School of Mathematical Sciences, Queen Mary and Westfield College, \\ Mile End Road, London E1 $4 N S$, UK
}

(Received 15 August 1997)

\begin{abstract}
We consider faithful finitary linear representations of (generalized) wreath products $A$ wr $\Omega H$ of groups $A$ by $H$ over (potentially) infinite-dimensional vector spaces, having previously considered completely reducible such representations in an earlier paper. The simpler the structure of $A$ the more complex, it seems, these representations can become. If $A$ has no non-trivial abelian normal subgroups, the conditions we present are both necessary and sufficient. They imply, for example, that for such an $A$, if there exists such a representation of the standard wreath product $A$ wr $H$ of infinite dimension, then there already exists one of finite dimension.
\end{abstract}

Keywords: finitary linear; wreath products

AMS 1991 Mathematics subject classification: Primary 20H20

Secondary 20E22; 20F29

If $V$ is a vector space over some field $F$, then $\operatorname{FGL}(V)$, the finitary general linear group over $V$, consists of all $F$-automorphisms $g$ of $V$ for which $\operatorname{dim}_{F} V(g-1)$ is finite. Over the past decade much has been learnt about such groups FGL $(V)$ and particularly about their subgroups (usually called finitary linear groups). For a summary of and introduction to work up to about 1994 , see [1].

Linear groups of finite degree are very 'narrow' groups; direct products and wreath products are rarely embeddable in them. Finitary linear groups do not superficially seem to be so narrow in that there are no comparable restrictions on their direct-product subgroups. Specifically, every direct product of finitary linear groups of the same ground-field characteristic is isomorphic to a finitary linear group (of that characteristic). However, the results of this present paper and of our earlier paper [7] on wreath products indicate clearly that there is some sort of, as yet ill-defined, narrowness about finitary linear groups.

In [7] we made a start on the classification of wreath products with faithful finitary representations. Primarily we were concerned there with wreath products having completely reducible such representations. Here we embark on the general case. Our results are reasonably complete if, for example, the base group is not soluble. The next step, it seems, will need to be the study of such representations where the image of the base 
group is unipotent and then, in the general case, the determination of the structure of the unipotent radical of the base group in such a representation. To do this it seems likely that some substantial new idea is required; $I$ feel the techniques of the present paper and of [7] have been pushed about as far as they will go.

Finitary linear, standard wreath products seem relatively rare. For example, it follows at once from the corollary below and the finite-dimensional case, that if $A$ and $H$ are nontrivial groups such that $A$ has no non-trivial abelian normal subgroups, then the standard wreath product $G=A$ wr $H$ is isomorphic to a finitary linear group of characteristic $p \geqslant 0$ if and only if $G$ is actually isomorphic to some linear group of finite degree and characteristic $p$. Thus the switch from finite-dimensional to infinite-dimensional spaces has not in this instance increased the range of examples. It pays, therefore, to be a little more general. First we explain our notation.

Let $A$ and $H$ be two groups, let $\Omega$ be a set and let $\sigma$ be a homomorphism of $H$ into the symmetric group $\operatorname{Sym}(\Omega)$. For each $\omega$ in $\Omega$ let $a \mapsto a_{\omega}$ be an isomorphism of $A$ onto the group $A_{\omega}$ and let $B$ be the direct product over $\omega$ in $\Omega$ of the copies $A_{\omega}$ of $A_{\omega}$ Then $H$ acts on $B$ by permuting the copies of $A$ via its action on $\Omega$. Specifically, for $a$ in $A$, $\omega$ in $\Omega$ and $h$ in $H$, let $\left(a_{\omega}\right)^{h}=a_{\omega h}$. We consider here the generalized wreath product $G=A$ wr $_{\Omega} H$ of $A$ by $H$ over $\Omega$, that is, the split extension $G=H[B=H B$ of $B$ by $H$. Throughout this paper $F$ denotes a field of characteristic $p \geqslant 0$ and $V$ a vector space over $F$. If $K$ is some subgroup of $\operatorname{FGL}(V)$, then $u(K)$ denotes the unipotent radical of $K$. The following summarizes our main conclusions here.

Theorem. Consider the group $G=H B=A \mathrm{wr}_{\Omega} H$ above.

(a) Suppose $H \sigma \neq\langle 1\rangle$ and assume $G$ is a subgroup of $\mathrm{FGL}(V)$. Then $A$ has a nilpotent normal subgroup $Q$ such that $A / Q$ is isomorphic to a completely reducible linear group of finite degree and characteristic $p$. Further, if $p=0$, then $Q$ is torsion-free and if $p>0$, then $Q$ has finite exponent a power of $p$. If $A$ is not nilpotent-by-abelian (e.g. if $A$ is not soluble), then $H \sigma \leqslant F \operatorname{Sym}(\Omega)$.

(b) If $A$ is isomorphic to a linear group of finite degree and characteristic $p$, if $H$ is isomorphic to a finitary linear group of characteristic $p$ and if $H \sigma \leqslant F \operatorname{Sym}(\Omega)$, then $G$ is isomorphic to some finitary linear group of characteristic $p$.

(c) Suppose $H \sigma=\langle 1\rangle$. Then $G$ is isomorphic to a finitary linear group of characteristic $p$ if and only if $A$ and $H$ are so isomorphic.

The main part of the theorem is (a). It strongly suggests that if $A$ is not soluble, then $G$ is isomorphic to a finitary linear group of characteristic $p$ if and only if $H$ is so isomorphic and if either $A$ is isomorphic to a linear group of finite degree and characteristic $p$ and $H \sigma \leqslant F \operatorname{Sym}(\Omega)$, or $A$ is isomorphic to a finitary linear group of characteristic $p$ and $H \sigma=\langle 1\rangle$. (If $A=\langle 1\rangle$, these conditions are trivially inadequate. They are also inadequate if $A$ is abelian and non-trivial, as is shown by the theorem of [6], Point 4(d) of [7] and also the result 2 below.) In particular, the following is immediate from the theorem. 
Corollary. With the notation as above, suppose $A$ has no non-trivial abelian normal subgroups. Then $G=A \mathrm{wr}_{\Omega} H$ is isomorphic to a finitary linear group of characteristic $p$ if and only if $H$ is isomorphic to a finitary linear group of characteristic $p$ and if either $A$ is isomorphic to a linear group of finite degree and characteristic $p$ and $H \sigma \leqslant F \operatorname{Sym}(\Omega)$, or $A$ is isomorphic to a finitary linear group of characteristic $p$ and $H \sigma=\langle 1\rangle$, or $A=\langle 1\rangle$.

It is clear from [7], and also from the present paper, that the case where the group $A$ (in $\left.G=A \operatorname{wr}_{\Omega} H\right)$ is torsion-free abelian, is critical and potentially complex. If the conditions on $\Omega, \sigma$ and $H$ required for faithfully representing $G$ depended upon the isomorphism type of $A$, then the situation could become very involved indeed. Fortunately, this is not the case.

Proposition. Suppose, for some torsion-free abelian group $A_{0} \neq\langle 1\rangle$, that the group $G_{0}=A_{0} \operatorname{wr}_{\Omega} H$ is isomorphic to a finitary linear group of characteristic $p \geqslant 0$. Then for any torsion-free abelian group $A$, the group $G=A \mathrm{wr}_{\Omega} H$ is isomorphic to a finitary linear group of characteristic $p$.

We prove the proposition in three steps. First we consider the case where the image of the base group $B_{0}$ of $G_{0}$ is completely reducible. This effectively settles the positivecharacteristic case. Then we assume the image of $B_{0}$ is unipotent (so necessarily here char $F=0$ ). Finally, we reduce the general characteristic-zero case to the previous situation.

$\S 1$. Consider $G=A \mathrm{wr}_{\Omega} H$. Suppose $\Omega$ has a system of imprimitivity $\Omega=\bigcup_{i \in I} \Omega_{i}$ for $H$ such that for each $i$ in $I$ the wreath product of $A$ by $N_{H}\left(\Omega_{i}\right) / C_{H}\left(\Omega_{i}\right)$ over $\Omega_{i}$ is isomorphic to a linear group of finite degree and characteristic $p \geqslant 0$ and such that for every $h$ in $H$ there is a cofinite subset $I(h)$ of $I$ with $h$ centralizing $\bigcup_{i \in I(h)} \Omega_{i}$. Assume further that $H$ is isomorphic to some finitary linear group of characteristic $p$. Then $G$ too is isomorphic to some finitary linear group of characteristic $p$.

Proof. Suppose first that $H$ is transitive on $I$. Pick $i$ in $I$. Set $\Sigma=\Omega_{i}$, set $N=N_{H}(\Sigma)$ and set $C=C_{H}(\Sigma)$. Let $T$ be a transversal of $N$ to $H$; whence $\Omega=\bigcup_{t \in T} \Sigma t$ is the given system of imprimitivity. Let $B_{\Sigma}=\left\langle A_{\omega}: \omega \in \Sigma\right\rangle$, so $B$ is the direct product of the $\left(B_{\Sigma}\right)^{t}$ over $t$ in $T$.

By hypothesis there is a field $F$ of characteristic $p$, a vector space $U$ over $F$ of finite dimension and an embedding of $(N / C) B_{\Sigma} \cong A \mathrm{wr}_{\Sigma}(N / C)$ into $\mathrm{GL}(U)$. Now $C\left\langle\left(B_{\Sigma}\right)^{t}\right.$ : $t \notin N\rangle$ is a normal subgroup of $N B$. Regard $U$ as a $B_{\Sigma}$-faithful FNB-module via the obvious map of $N B$ onto $(N / C) B_{\Sigma}$. Then $V=U \otimes_{\mathrm{FNB}} \mathrm{FG}=\oplus_{T} U \otimes t$ is a $B$-faithful FG-module.

Clearly, $B_{\Sigma} \leqslant \mathrm{FGL}(V)$, so $B \leqslant \mathrm{FGL}(V)$. If $h \in H$, there is, by hypothesis, a cofinite subset $S$ of $T$ such that $h$ centralizes $\bigcup_{s \in S} \Sigma s$. If $s \in S$, then $s h=n s$ for some $n \in N$ and for any $\sigma$ in $\Sigma$ we have $\sigma s=(\sigma s) h=(\sigma n) s$, so $n \in C$. Thus $h$ centralizes $\oplus_{s \in S} U \otimes s$. The latter has cofinite dimension in $V$. Thus $G$ acts finitarily on $V$. (In particular, if $H \leqslant \mathrm{FGL}(W)$ for some vector space $W$ over $F$, then $G$ embeds into $\mathrm{FGL}(V \oplus W)$.) 
Suppose now that $H$ is intransitive on $I$. For a large enough field $F$ of characteristic $p$, for each orbit $J$ of $H$ in $I$ we can construct as above a finitary $F H\left\langle A_{\omega}: \omega \in \bigcup_{i \in J} \Omega_{i}\right\rangle$ module $V_{J}$ that is faithful for $\left\langle A_{\omega}: \omega \in \bigcup_{i \in J} \Omega_{i}\right\rangle$ and trivial for $C_{H}\left(\bigcup_{i \in J} \Omega_{i}\right)$. Set $V=$ $\oplus_{j} V_{J}$. Then $V$ is an $F G$-module that is clearly $B$-faithful and $C_{H}(\Omega)$-trivial. Moreover $B$ embeds into $\mathrm{FGL}(V)$. Let $h \in H$. By construction $h$ acts trivially on $V_{J}$ for all but a finite number of $J$. Consequently, $G$ acts finitarily on $V$. Finally, we may also choose $F$ sufficiently large so that, for some vector space $W$, the group $H$ embeds into FGL $(W)$. Consequently, $G$ will then embed into $\mathrm{FGL}(V \oplus W)$.

$\S 2$. Again consider $G=A \mathrm{wr}_{\Omega} H$ and suppose $H$ is isomorphic to a finitary linear group of characteristic $p \geqslant 0$. Then under any one of the following four conditions, $G$ is also isomorphic to some finitary linear group of characteristic $p$.

(a) $A$ is isomorphic to a linear group of finite degree and characteristic $p$ and $H \sigma \leqslant$ $F \operatorname{Sym}(\Omega)$.

(b) $A$ is isomorphic to a finitary linear group of characteristic $p$ and $H \sigma=\langle 1\rangle$.

(c) $A=\langle 1\rangle$.

(d) $\Omega$ has a system of imprimitivity $\Omega=\bigcup_{i \in I} \Omega_{i}$ for $H$ such that every $h$ in $H$ there is a cofinite subset $I(h)$ of $I$ with $h$ centralizing $\bigcup_{i \in I(h)} \Omega_{i}$. For each $i$ in $I$, either $p>0$, the group $A$ is abelian with its periodic subgroup a $p$-group of finite exponent and $N_{H}\left(\Omega_{i}\right) / C_{H}\left(\Omega_{i}\right)$ is finite; or $p=0$, the group $A$ is torsion-free abelian and $N_{H}\left(\Omega_{i}\right) / C_{H}\left(\Omega_{i}\right)$ contains an abelian normal subgroup $L$ of finite index such that $\Omega_{i}$ contains only a finite number of isomorphism types of $L$-orbit and each $L / C_{L}(\omega)$ for $\omega$ in $\Omega_{i}$ is torsion-free; or $p>0$, the group $A$ is an abelian $p$-group of finite exponent and $N_{H}\left(\Omega_{i}\right) / C_{H}\left(\Omega_{i}\right)$ contains a subgroup $L$ as in the previous case.

I do not know of an example where the group $G$ above is isomorphic to a finitary linear group, that is not covered by one of the four cases in 2 . Thus the converse to 2 is open. If this converse holds, it is not difficult to deduce the converse to 4 of [7] (settling the finitary linearity of $G$ with $B$ completely reducible) and to confirm the speculation in the introduction to [7] concerning the finitary representability of $G$ as a completely reducible group. Note that parts (b) and (c) of the theorem are easy consequences of (a) and (b) of 2 .

Proof. (a) By the enlargement of fields we may assume here that $A \leqslant \mathrm{GL}(U)$ and $H \leqslant$ FGL $(W)$, where $U$ and $W$ are vector spaces over the same field $F$ of characteristic $p$ with $\operatorname{dim}_{F} U$ finite. Let $V=\oplus_{\omega \in \Omega} U_{\omega}$, where each $U_{\omega}$ is a copy of $U$. Then $A \operatorname{wr}_{\Omega} H \sigma$ embeds into $\mathrm{GL}(V)$ in the obvious way. Moreover, $A \mathrm{wr}_{\Omega} H \sigma$ embeds, in fact, into FGL $(V)$, since $H \sigma \leqslant F \operatorname{Sym}(\Omega)$. Finally, $G=A \operatorname{wr}_{\Omega} H$ embeds into $\mathrm{FGL}(V \oplus W)$. 
(b) Here we may assume that $A \leqslant \mathrm{FGL}(U)$ and $H \leqslant \mathrm{FGL}(W)$, where $U$ and $W$ are vector spaces over the field $F$ of characteristic $p$. Since $H \sigma=\langle 1\rangle$, we have $G \cong A^{(\Omega)} \times H$ and hence $V=U^{(\Omega)} \oplus W$ can be made into a $G$-faithful finitary $F G$-module.

(c) Here $G=H$, so the claim is obvious.

(d) The theorem of [6] shows that the hypotheses of 1 are satisfied.

$\S 3$. Suppose $G=H B=A \mathrm{wr}_{\Omega} H$ is a subgroup of $\mathrm{FGL}(V)$ with $B$ unipotent and $H \sigma \neq\langle 1\rangle$.

(a) If $p=0$, then $A$ is torsion-free nilpotent.

(b) If $p>0$, then $A$ is a nilpotent $p$-group of finite exponent.

Proof. By hypothesis there exists $h$ in $H$ and $\omega$ in $\Omega$ with $\omega h \neq \omega$. Let $C=A_{\omega} \times$ $A_{\omega h} \leqslant B$ and let $N$ he the kernel of the product map of $C$ onto $A / A^{\prime}$ (that is of the map $\left.a_{\omega} a_{\omega h}^{\prime} \mapsto a a^{\prime} A^{\prime}\right)$. Now there is a $G$-invariant series $\left\{\left(\Lambda_{\sigma}, V_{\sigma}\right): \sigma \in \Sigma\right\}$ of subspaces of $V$ stabilized by $B$ (see, for example, $[2,2.2 \mathrm{a}]$ ). Let $r=\operatorname{dim}_{F}[V, h]<\infty$. Then there are $r$ factors

$$
\Lambda_{\sigma(i)} / V_{\sigma(i)}, \quad \text { with } \sigma(1) \leqslant \sigma(2) \leqslant \cdots \leqslant \sigma(r),
$$

of this series, not necessarily all distinct, such that $h$ centralizes the $r+1$ factors

$$
V_{\sigma(1)}, V_{\sigma(i+1)} / \Lambda_{\sigma(i)} \text { for } 1 \leqslant i<r \text { and } V / \Lambda_{\sigma(r)} \text {. }
$$

If $a \in A$, then $a_{\omega}$ and $a_{\omega h}$ induce the same map on these latter $r+1$ factors and hence $N$ centralizes these $r+1$ factors. Also $B \geqslant N$ centralizes the former $r$ factors. Thus $N$ stabilizes a series of subspaces in $V$ (running from $\{0\}$ to $V$ ) of length $2 r+1$. Therefore, $N$ is a nilpotent group of class at most $2 r$. Further $N$ is torsion-free if $p=0$ and of exponent dividing $p^{2 r}$ if $p>0$. If $a \in A$, then $a_{\omega} a_{\omega h}^{-1} \in N$, so $N$ projects onto $A$ via its first component. Thus $A$ is nilpotent of class at most $2 r$ and, if $p>0$, of exponent dividing $p^{2 r}$. Moreover, if $a$ has finite order $q$, then so does $a_{\omega} a_{\omega h}^{-1} \in N$. Therefore, $A$ is torsion-free if $p=0$. The proof is complete.

$\S 4$. Let $G=H B=A \mathrm{wr}_{\Omega} H$ be a subgroup of $\mathrm{FGL}(V)$ and suppose $\omega h \neq \omega$ for some $\omega \in \Omega$ and $h \in H$.

(a) If $p=0$, then the unipotent radical $u\left(A_{\omega}\right)$ is torsion-free nilpotent.

(b) If $p>0$, then $u\left(A_{\omega}\right)$ is a nilpotent $p$-group of finite exponent.

If $p>0$, then $u\left(A_{\omega}\right)$ is canonical in that $u\left(A_{\omega}\right)=O_{p}\left(A_{\omega}\right)=O_{p}(A)_{\omega}$. If $p=0$ and $\lambda$ and $\mu$ lie in different $H$-orbits of $\Omega$, then $u\left(A_{\lambda}\right)$ and $u\left(A_{\mu}\right)$ need not even be isomorphic, as simple examples show. This is a substantial extra complication in the characteristic-zero case.

Proof. Apply 3 with $A$ and $\Omega$ replaced by $u\left(A_{\omega}\right)$ and $\omega H$. Note that $u\left(A_{\omega}\right)^{k}=u\left(A_{\omega k}\right)$ for every $k$ in $H$ and that $u\left(A_{\omega}\right) w_{\omega} H \cong\left\langle u\left(A_{\omega}\right), H\right\rangle \leqslant G$. 
$\S 5$. Suppose $G=H B=A \mathrm{wr}_{\Omega} H$ is a subgroup of $\mathrm{FGL}(V)$, where $p>0$. If $A$ is not an extension of a nilpotent $p$-group of finite exponent by a torsion-free abelian group, then $H \sigma \leqslant F \operatorname{Sym}(\Omega)$. Moreover, unless $H \sigma=\langle 1\rangle$, the subgroup $O_{p}(A)$ of $A$ is nilpotent of finite exponent and $A / O_{p}(A)$ is isomorphic to a completely reducible linear group of finite degree over the algebraic closure of $F$.

Proof. Clearly, we may assume that $H \sigma \neq\langle 1\rangle$. Also $O_{p}(B)=\times_{\Omega} O_{p}\left(A_{\omega}\right)$ is the unipotent radical of $B$, we have $G_{1}=G / O_{p}(B)$ isomorphic to $\left(A / O_{p}(A)\right.$ wr $\Omega H$ and if $W$ is the direct sum of the $F G$-composition factors of $V$, then $W$ is a finitary $B / O_{p}(B)$ faithful completely reducible $F G_{1}$-module. Moreover $O_{p}(A)$ is nilpotent of finite exponent a power of $p$ by $4(\mathrm{~b})$, so $A / O_{p}(A)$ is not torsion-free abelian. Therefore, $H \sigma \leqslant F \operatorname{Sym}(\Omega)$ and $A / O_{p}(A)$ is isomorphic to a completely reducible linear group of finite degree over the algebraic closure of $F$ by 2 of [7].

To produce a characteristic-zero version of 5 , we need some further preparation.

$\S 6$. For $G=H B=A \mathrm{wr}_{\Omega} H$, suppose $W$ is a finitary $F G$-module such that the base group $B$ of $G$ acts completely reducibly on $W$. With $K$ the kernel of the natural map of $B$ into $\mathrm{FGL}(W)$ and $K_{\omega}$ the natural projection of $K$ into $A_{\omega}$, assume that $A_{\omega} / K_{\omega}$ is non-abelian for every $\omega$ in $\Omega$. Then $H \sigma \leqslant F \operatorname{Sym}(\Omega)$.

Proof. We extract what we can from the proof of Lemma 2 in [7]. Clearly, we may assume that $H$ has no fixed points in $\Omega$. We may also assume $F$ is algebraically closed, for if not let $E$ be the algebraic closure of $F$ and replace $F$ and $W$ by $E$ and the sum of the $E G$-composition factors of $E \otimes_{F} W$.

If $U$ is an irreducible $F B$-submodule of $W$ with $\operatorname{dim}_{F} U$ infinite, then since $W$ is finitary, $U$ is an FG-submodule of $W$. By Lemma 6 of [4], at most one $A_{\omega}$ acts nontrivially on $U$. But now $H$ has no fixed points in $\Omega$ and if $A_{\omega}$. acts non-trivially on $U$, so does $A_{\omega h}$ for every $h$ in $H$. Thus every $A_{\omega}$ acts trivially on $U$ and $\operatorname{dim}_{F} U=1$. This contradiction shows that $W$ is a direct sum of finite $F$-dimensional irreducible $F B$-modules. Let $\left\{W_{i}: i \in 1\right\}$ be the set of non-zero, non- $B$-trivial, $F B$-homogeneous components of $W$, so $W=C_{W}(B) \oplus\left(\oplus_{I} W_{i}\right)$ and set $W_{0}=\oplus_{I} W_{i}$. By finitariness, each $\operatorname{dim}_{F} W_{i}$ is finite.

For $i \in I$ let $\Omega_{i}=\left\{\omega \in \Omega: A_{\omega}\right.$ does not act as a group of scalars on $\left.W_{i}\right\}$. If $U$ is an irreducible $F B$-submodule of $W_{i}$, there is, since $\operatorname{dim}_{F} U$ is finite, a finite subset $\Xi_{i}$ of $\Omega_{i}$ such that $U$ is irreducible as $F\left\langle A_{\omega}: \omega \in \Xi_{i}\right\rangle$-module. By Schur's Lemma (recall $F$ is now algebraically closed) $A_{\omega}$ acts as a group of scalars on $U$ and hence on $W_{i}$ for every $\omega$ in $\Omega \backslash \Xi_{i}$. Therefore, $\Omega_{i}=\Xi_{i}$, which is finite.

Since $B$ is normal in $G$, so $G$ permutes the $W_{i}$ and permutes them finitarily. Let $h \in H \backslash\langle 1\rangle$. For some finite subset $J$ of $I$, the element $h$ centralizes $W_{i}$ for each $i$ in $I \backslash J$. Let $\omega \in \Omega \backslash \Omega_{J}$ for $\Omega_{J}=\bigcup_{j \in J} \Omega_{j}$. By hypothesis there is some $a \in A^{\prime}$ (and probably depending on the choice of $\omega$ ) such that $a_{\omega} \notin K_{\omega}$. If $j \in J$, then $\omega \notin \Omega_{J}$ and $A_{\omega}$ is scalar on $W_{j}$. Thus for all $j \in J$ the element $a_{\omega}$ acts as 1 on $W_{j}$ and $a_{\omega h}=\left(a_{\omega}\right)^{h}$ acts as $a_{\omega}$ on 
$W_{j}$. Let $i \in I \backslash J$. Then $h$ centralizes $W_{i}$ and again $a_{\omega h}$ acts as $a_{\omega}$ on $W_{i}$. Consequently, $a_{\omega}$ and $a_{\omega h}$ have the same action on $W_{0}$ and hence on $W$. Therefore, $a_{\omega h} a_{\omega}^{-1} \in K$ and so if $\omega h \neq \omega$, then $a_{\omega} \in K_{\omega}$, which is false. Therefore, $\omega h=\omega$. We have now proved that the cofinite subset $\Omega \backslash \Omega_{J}$ of $\Omega$ consists of fixed points of $h$. Consequently, $h$ acts finitarily on $\Omega$, which means that $H$ does too.

If $K$ is a group, then $\gamma^{3} K$ denotes $[K, K, K]$, the third term of the lower central series of $K$. We have the following corollary of 6 .

$\S 7$. For $G=H B=A \operatorname{wr}_{\Omega} H$, suppose $W$ is a finitary $F G$-module such that the base group $B$ of $G$ acts completely reducibly on $W$. Suppose $\gamma^{3} A_{\omega}$ acts non-trivially on $W$ for every $\omega$ in $\Omega$ (e.g. if $H$ is transitive on $\Omega$ and $\left[W, \gamma^{3} B\right] \neq\{0\}$ ). Then $H \sigma \leqslant F \operatorname{Sym}(\Omega)$.

Proof. Let $K$ and $K_{\omega}$ be as in 6 . Then $\left[A_{\omega}, K_{\omega}\right]=\left[A_{\omega}, K\right] \leqslant A_{\omega} \cap K \leqslant K_{\omega}$ for any $\omega$ in $\Omega$. By hypothesis $\gamma^{3} A_{\omega}$ is not in $K$, so $A_{\omega}^{\prime}$ does not lie in $K_{\omega}$. Now 6 applies.

$\S 8$. Suppose $G=H B=A \operatorname{wr}_{\Omega} H$ is a subgroup of $\operatorname{FGL}(V)$ and that there is some $\omega$ in $\Omega$ and $h$ in $H$ with $\omega h \neq \omega$ and $A_{\omega}$ locally nilpotent and unipotent-by-abelian. Then $A$ is nilpotent.

Proof. We may assume $F$ is algebraically closed. Then, being locally nilpotent, $A_{\omega}$ has a Jordan decomposition $A_{\omega} \leqslant\left(A_{\omega}\right)_{u} \times\left(A_{\omega}\right)_{d} \leqslant \mathrm{FGL}(V)$; see $\S 2$ of [3], especially 2.3 . Clearly, $\left(\left(A_{\omega}\right)_{u}\right)^{k}=\left(A_{\omega}\right)_{u}$ for every $k$ in $H$, and similarly with $d$ in place of $u$. Also the four subgroups $\left(A_{\omega}\right)_{u},\left(A_{\omega}\right)_{d},\left(A_{\omega h}\right)_{u}$ and $\left(A_{\omega h}\right)_{d}$ centralize each other. By hypothesis $A_{\omega}^{\prime} \leqslant\left(A_{\omega}\right)_{u}$, so $\left(A_{\omega}\right)_{d}$ is abelian.

Consider $\bar{G}=\left\langle G,\left(A_{\omega}\right)_{u}\right\rangle$. There is a $\bar{G}$-invariant series $\left\{\left(\Lambda_{\sigma}, V_{\sigma}\right): \sigma \in \Sigma\right\}$ of subspaces of $V$ stabilized by $u(\bar{G})$. Let $r=\operatorname{dim}_{F}[V, h]<\infty$. Then there are $r$ factors

$$
\Lambda_{\sigma(i)} / V_{\sigma(i)}, \quad \text { with } \sigma(1) \leqslant \sigma(2) \leqslant \cdots \leqslant \sigma(r),
$$

of this series, not necessarily all distinct, such that $h$ centralizes the $r+1$ factors

$$
V_{\sigma(1)}, V_{\sigma(i+1)} / \Lambda_{\sigma(i)} \text { for } 1 \leqslant i<r \text { and } V / \Lambda_{\sigma(r)} .
$$

If $x \in\left(A_{\omega}\right)_{u}$, then $x$ and $x^{h}$ induce the same map on these latter $r+1$ factors and, since they lie in $u(\bar{G})$ centralize the former $r$ factors. Then the subgroup $D_{0}$, generated by the $x^{-1} x^{h}$ for $x \in\left(A_{\omega}\right)_{u}$ stabilizes a series in $V$ of length $2 r$ and therefore is nilpotent (of class at most $2 r$ ). Hence so too is the subgroup $\left\langle D_{0},\left(A_{\omega}\right)_{d},\left(A_{\omega h}\right)_{d}\right\rangle$. Consequently, the subgroup $D$ of $A_{\omega} \times A_{\omega h} \leqslant B$ generated by all $a_{\omega}^{-1} a_{\omega h}$ for $a \in A$ is nilpotent. But $D$ projects onto $A$, say via the first factor. Therefore $A$ is nilpotent.

$\S 9$. Suppose $G=H B=A \operatorname{wr}_{\Omega} H$ is a subgroup of $\mathrm{FGL}(V)$, where $F$ is algebraically closed of characteristic zero.

(a) If $H \sigma \notin F \operatorname{Sym}(\Omega)$, then $A$ is nilpotent-by-abelian. 
(b) If $H \sigma \neq\langle 1\rangle$ and $F$ has sufficiently large transcendence degree (e.g. $\operatorname{tr} \operatorname{deg} F \geqslant|A|$ suffices), then $A$ is an extension of a torsion-free nilpotent group by a completely reducible linear group over $F$ of finite degree.

Part (a) of the theorem follows at once from 5 and 9.

Proof. (a) Clearly, we may assume that $H$ has no fixed points in $\Omega$. Let $W$ be the sum of the $B$-non-trivial $F G$-composition factors of $V$. Then $W$ is a $B / u(B)$-faithful, completely $F B$-reducible, finitary $F G / u(B)$-module (but here $G / u(B)$ need not be a wreath product, unlike the case in 5). Let $\omega \in \Omega$ and denote the projection of $u(B)$ into $A_{\omega}$ by $K_{\omega}$. Certainly $K_{\omega}$ is locally nilpotent and $\left(K_{\omega}\right)^{h}=K_{\omega h}$ for all $h \in H$. Also $\left[A_{\omega}, u(B)\right] \leqslant A_{\omega} \cap u(B) \leqslant u\left(K_{\omega}\right)$, so $K_{\omega}$ is unipotent-by-abelian. Apply 8 to the subgroup $\left\langle H, K_{\omega}\right\rangle \cong K_{\omega} \mathrm{wr}_{\omega H} H$ of $G$. This yields that $K_{\omega}$ is nilpotent. Since by hypothesis $H \sigma \neq \operatorname{Sym}(\Omega)$. Point 6 implies that $A_{\omega} / K_{\omega}$ is abelian for some $\omega$ in $\Omega$. Therefore $A \cong A_{\omega}$ is nilpotent-by-abelian.

(b) Suppose $\omega h \neq \omega$ for some $\omega \in \Omega$ and $h \in H$. With $W$ as in the proof of (a), we have $W=\oplus_{i \in I} W_{i}$, where the $W_{i}$ are the $F B$-homogeneous components of $W$ and each $\operatorname{dim}_{F} W_{i}$ is finite, cf. the proof of 6 . Now $\operatorname{dim}_{F}[W, h]$ is finite, so for some finite subset $J$ of $I$, we have $[W, h] \leqslant X=\oplus_{j \in J} W_{j}$ and $Y=\oplus_{i \notin J} W_{i} \leqslant C_{W}(h)$. Let $\phi$ be the induced map of $G$ into $\operatorname{FGL}(W)$. Then $B \cap \operatorname{ker} \phi=u(B)$ and, just as we have seen in the proof of (a), the projection $K_{\omega}$ of $u(B)$ into $A_{\omega}$ is nilpotent.

Let $K$ (respectively $L$ ) be the kernel of the action of $C=A_{\omega} \times A_{\omega h} \leqslant B$ on $X$ (respectively $Y$ ). Then $K \cap L=\left.\operatorname{ker} \phi\right|_{C}=C \cap u(B)$. Denote by $N$ the normal subgroup of $C$ generated by all the elements $a_{\omega}^{-1} a_{\omega h}$ for $a \in A$. Then $N \leqslant L$. Also $C / N \cong A / A^{\prime}$ is abelian, so $[K, C] \leqslant K \cap L \leqslant \operatorname{ker} \phi$ and $K \phi \leqslant \zeta_{1}(C \phi)$, the centre of $C \phi$. The map $\psi: a_{\omega} \phi \mapsto\left(a_{\omega}^{-1} a_{\omega h}\right) \phi$ of the centre $\zeta_{1}\left(A_{\omega} \phi\right)$ of $A_{\omega} \phi$ into $N \phi$ is a homomorphism, since $\zeta_{1}\left(A_{\omega} \phi\right) \leqslant \zeta_{1}(C \phi)$, and $N \phi$ acts faithfully on $X$, since $N \phi$ acts faithfully on $W$ and trivially on $Y$. Therefore $\psi$ determines a homomorphism of $\zeta_{1}\left(A_{\omega} \phi\right)$ into $\mathrm{GL}(X)$ with kernel

$$
S=\left\{a_{\omega} \phi \in \zeta_{1}\left(A_{\omega} \phi\right): a \in A \& a_{\omega}^{-1} a_{\omega h} \in B \cap \operatorname{ker} \phi=u(B)\right\} .
$$

Since $\omega h \neq \omega$, this yields that $S \leqslant \zeta_{1}\left(A_{\omega} \phi\right) \cap K_{\omega} \phi$. Also $K \phi \leqslant \zeta_{1}(C \phi)$, so $\left(A_{\omega} \phi \cap K \phi\right) \leqslant$ $\zeta_{1}\left(A_{\omega} \phi\right)$. Thus $\left(A_{\omega} \phi \cap K \phi\right) /(S \cap K \phi)$ is isomorphic to an (abelian) subgroup of $\mathrm{GL}(X)$. Consequently, its maximal periodic subgroup $Z /(S \cap K \phi)$ has finite rank, $r$ say; necessarily $r \leqslant \operatorname{dim}_{F} X<\infty$.

Choose the normal subgroup $M$ of $A_{\omega} \phi$ maximal subject to

$$
M \cap Z\left(A_{\omega} \phi \cap L \phi\right)=(S \cap K \phi)\left(A_{\omega} \phi \cap L \phi\right) .
$$

Since $Z\left(A_{\omega} \phi \cap L \phi\right) /(S \cap K \phi)\left(A_{\omega} \phi \cap L \phi\right) \cong Z /(S \cap K \phi)$ is periodic abelian of finite rank $r$, so too is $A_{\omega} \phi / M$ (recall that $A_{\omega} \phi /\left(A_{\omega} \phi \cap L \phi\right)$ is abelian). In particular $A_{\omega} / M$ is isomorphic to a completely reducible (even diagonal) subgroup of $\operatorname{GL}(r, F)$.

Let $P$ be the maximal periodic subgroup of the nilpotent group $K_{\omega}$. Then $P$ wr $_{\Omega} H \leqslant$ $G \leqslant \mathrm{FGL}(V)$ and the base group of $P \mathrm{wr}_{\Omega} H$ is periodic and hence unipotent-free (recall 
char $F=0$ here). It follows from 2 of [7] that $P$ is isomorphic to a linear group of finite degree over $F$. Hence so too is $P \phi \cong P$ and thus the abelian group $P \phi \cap K \phi \leqslant \zeta_{1}(C \phi)$ has finite rank, $s$ say. Choose a normal subgroup $M_{1}$ of $A_{\omega} \phi$ maximal subject to

$$
M_{1} \cap(P \phi \cap K \phi)\left(A_{\omega} \phi \cap L \phi\right)=A_{\omega} \phi \cap L \phi .
$$

Then $A_{\omega} \phi / M_{1}$ is periodic abelian of rank $s$ and therefore is isomorphic to a completely reducible subgroup of $\mathrm{GL}(s, F)$.

Let $T$ be the subgroup of $A_{\omega} \phi$ such that $T /\left(A_{\omega} \phi \cap L \phi\right)$ is the maximal periodic subgroup of the abelian group $A_{\omega} \phi /\left(A_{\omega} \phi \cap L \phi\right)$. Then $A_{\omega} \phi / T$ is a torsion-free abelian group with cardinality at most $|A|$. Assuming this is at most $\operatorname{tr} \operatorname{deg} F$, for example, $A_{\omega} \phi / T$ is isomorphic to a (trivially irreducible) subgroup of $F^{*}$.

Clearly, $A_{\omega} \phi / K \phi$ embeds into $\mathrm{GL}(X)$ and, since $X$ is completely reducible as $F B$ module and $A_{\omega}$ is normal in $B$, the $F A_{\omega}$-module $X$ is also completely reducible. Set $Q_{0}=M_{1} \cap M \cap T \cap K \phi$. Then $Q_{0}$ is a normal subgroup of $A_{\omega} \phi$ and $A_{\omega} \phi / Q_{0}$ is isomorphic to a completely reducible subgroup of $\operatorname{GL}\left(r+s+\operatorname{dim}_{F} X, F\right)$.

Let $Q$ denote the inverse image in $A$ of $Q_{0}$ under the map $a \mapsto a_{\omega} \phi$. Then $Q$ is a normal subgroup of $A$ and $A / Q \cong A_{\omega} \phi / Q_{0}$. Now $K \phi \cap L \phi=\langle 1\rangle$, so $T \cap K \phi$ is periodic and so $T \cap K \phi \leqslant Z$. Also

$$
M \cap Z \leqslant(S \cap K \phi)\left(A_{\omega} \phi \cap L \phi\right) \cap K \phi=S \cap K \phi .
$$

Therefore $Q_{0} \leqslant S \cap K \phi \leqslant \zeta_{1}\left(A_{\omega} \phi\right) \cap K_{\omega} \phi$. Hence, with $Q_{\omega}=\left\{a_{\omega}: a \in Q\right\} \triangleleft A_{\omega}$, we have $Q_{\omega} \leqslant A_{\omega} \cap K \phi \phi^{-1}=A_{\omega} \cap K_{\omega} u(B)=K_{\omega}$. In particular $Q$ is nilpotent. Finally, the maximal periodic subgroup of $Q_{\omega}$ is $Q_{\omega} \cap P$ and

$$
Q_{\omega} \cap P \cong\left(Q_{\omega} \cap P\right) \phi \leqslant M_{1} \cap P \phi \cap K \phi \leqslant L \phi \cap K \phi=\langle 1\rangle,
$$

so $Q$ is also torsion-free. The proof is now complete

$\S 10$. Consider the following example. Let $F=k(x)$, where $k$ is the rationals if $p=0$ and the field of $p$ elements otherwise and $x$ is an indeterminate over $k$. Let $V$ be a vector space over $F$ with the countable basis $\left\{v_{i}: i=1,2, \ldots\right\}$. We compute matrices only with respect to this basis. Let $h=\operatorname{diag}(x, 1,1, \ldots) \in \mathrm{FGL}(V)$. Let $A$ be the set of all matrices $\left(\alpha_{i j}\right)$ in FGL $(V)$ such that $\alpha_{i i}=1, \alpha_{i j}=0$ if $i \neq j \neq 1$ and $\alpha_{i 1} \in k$; this for all $i, j=1,2, \ldots$. Note that if $a=\left(\alpha_{i j}\right) \in A$, then $a^{h}=\left(\beta_{i j}\right)$, where $\beta_{i i}=1, \beta_{i j}=0$ if $i \neq j \neq 1$ and $\beta_{i 1}=\alpha_{i 1} x$ if $i \neq 1$. Thus $G=\langle h, A\rangle \leqslant \mathrm{FGL}(V)$ is isomorphic to the standard wreath product of $A$ by the infinite cyclic group $H=\langle h\rangle$.

However, $A$ is uncountable, so $A$ is not isomorphic to any linear group of finite degree over the countable field $F$, or even over the algebraic closure of $F$. However, $A$ is isomorphic to a linear group of finite degree ( 1 if $p=0$ and 2 otherwise) over some extension field of $F$, since $A$ is torsion-free abelian if $p=0$ and is an elementary abelian $p$-group if $p>0$. Thus when searching for finite-dimensional representations of the group $A$, in analogues of the above one must allow the possibility of substantially extending the ground field.

We now prove the proposition. The following is an easy consequence of 2 and 4 of [7]. 
$\S 11$. With $G=A \operatorname{wr}_{\Omega} H$ as above, suppose $H \sigma \leqslant F \operatorname{Sym}(\Omega)$. Then $G$ is isomorphic to a finitary linear group of characteristic $p \geqslant 0$ with the image of its base group (respectively of $G$ ) completely reducible if and only if $H$ is isomorphic to a finitary linear group of characteristic $p$ (respectively with $\operatorname{ker} \sigma$ completely reducible) and either $A$ is isomorphic to a completely reducible linear group of finite degree and characteristic $p$ (respectively with $H$ completely reducible if $A=\langle 1\rangle$ ), or $H \sigma=\langle 1\rangle$ and $A$ is isomorphic to a completely reducible finitary linear group of characteristic $p$.

$\S 12$. Suppose $G_{0}=A_{0} \mathrm{wr}_{\Omega} H \leqslant \operatorname{FGL}(V)$, with $A_{0} \neq\langle 1\rangle$ and the base group $B_{0}$ of $G_{0}$ (respectively $G_{0}$ itself) completely reducible. If $A$ is any torsion-free abelian group, then $G=A \operatorname{wr}_{\Omega} H$ is isomorphic to a finitary linear group of characteristic $p(p=\operatorname{char} F)$ with the image of its base group $B$ completely reducible (with $G$ itself completely reducible, provided $A \neq\langle 1\rangle)$.

Proof. If $A_{0}$ is not torsion-free abelian, then $H \sigma \leqslant F \operatorname{Sym}(\Omega)$ by 2 of [7]. Now $A$, being torsion-free abelian, is certainly isomorphic to a linear group of degree 1 and characteristic $p$, and as such is clearly irreducible. Also if $G_{0}$ is completely reducible, then so is its normal subgroup $\operatorname{ker} \sigma$ (the extended Clifford Theorem). The conclusions now follow from 11 above.

Assume, therefore, that $A_{0}$ is torsion-free abelian. We concentrate first on the case where $G_{0}$, is not necessarily completely reducible. For this we may also assume that $A_{0}=\langle a\rangle$ is infinite cyclic.

Suppose $G_{0}=\langle a\rangle \operatorname{wr}_{\Omega} H \leqslant \mathrm{FGL}(V)$ with $B_{0}$ completely reducible. We may assume $F$ is algebraically closed. (Otherwise for $F^{\wedge}$ the algebraic closure of $F$, replace $V$ by the direct sum of $F^{\wedge} \otimes_{F} V$, with $G$ acting via $G \rightarrow H$, and the direct sum of the $F G$ composition factors of $F^{\wedge} \otimes_{F} V$, with $G$ acting naturally.) Let $\left\{V_{i}: i \in I\right\}$ be the set of non-zero, $B_{0}$-non-trivial, $F B_{0}$-homogeneous components of $V$, so $V=C_{V}\left(B_{0}\right) \oplus V_{0}$ for $V_{0}=\oplus_{I} V_{i}$. Then $B_{0}$ acts on each $V_{i}$ as a group of scalars (since $F$ is algebraically closed) and $H$ permutes the $V_{i}$ and hence acts on $I$ via $V_{i h}=V_{i} h$ for $h$ in $H$. Further, by finitariness, each $\operatorname{dim}_{F} V_{i}$ is finite.

Assume first that $H$ is transitive on $\Omega$; say $\Omega=\pi H=\pi T$ for $T$ a right transversal of $N_{H}(\pi)$ to $H$. Now $a_{\pi}$, acts on $V_{i}$ as a scalar, say as $\eta_{i}$. Choose elements $\xi_{i}$ for $i$ in $I$ algebraically independent over $F$ subject to $\xi_{i}=\xi_{j}$ whenever $\eta_{i}=\eta_{j}$, and $\xi_{i}=1$ whenever $\eta_{i}=1$. Set $R=F\left[\xi_{i}, \xi_{i}^{-1}: i \in I\right] \leqslant E$, where $E$ is some algebraically closed extension field of $F$ of large transcendence degree over $F\left(\operatorname{tr} \operatorname{deg} E / F=\left(\operatorname{dim}_{F} V\right)(|\Omega|)(\operatorname{rank} A)\right.$ will suffice). Let $U_{i}=E \otimes_{F} V_{i}$ and $U_{0}=E \otimes_{F} V_{0}=\oplus_{I} U_{i}$ after the obvious identifications. Let $x_{\pi} \in \mathrm{GL}\left(U_{0}\right)$ act on $U_{i}$ for each $i$ in $I$ as the scalar $\xi_{i}$. The $F$-algebra homomorphism $\theta$ of $R$ to $F$ defined by $\theta: \xi_{i} \mapsto \eta_{i}$ for each $i$ defines a group homomorphism $\phi$ of $\left\langle H, x_{\pi}\right\rangle$ onto $G_{0}=\left\langle H, a_{\pi}\right\rangle$. Then $x_{\pi} \phi=a_{\pi}$ and $\left(x_{\pi}\right)^{h} \phi=\left(a_{\pi}\right)^{h}=a_{\pi h}$. Note that $\left(x_{\pi}\right)^{h}$ acts on $U_{i h}$ as $\xi_{i}$ for each $i$ and hence commutes with $x_{\pi}$. Thus $\phi$ restricts to an isomorphism of $\left\langle\left(x_{\pi}\right)^{t}: t \in T\right\rangle$ onto $B_{0}=\left\langle a_{\pi t}: t \in T\right\rangle$. Suppose $\pi h=\pi$ for some $h \in H$. Then $a_{\pi h}=a_{\pi}$, and so $\eta_{i h}=\eta_{i}$ for each $i$. Hence $\xi_{i h}=\xi_{i}$ for each $i$ and thus $\left(x_{\pi}\right)^{h}=x_{\pi}$. Therefore, $\phi$ is an isomorphism of $\left\langle H, x_{\pi}\right\rangle$ onto $G_{0}$. 
Let $D$ denote the subgroup of all elements of FGL $\left(U_{0}\right)$ that act as scalars on each $U_{i}$. For $d$ in $D$ write $d=\operatorname{diag}\left(\delta_{i}: i \in I\right)$, where $d$ acts on each $U_{i}$ as $\delta_{i}$. Note that $x_{\pi}$ and $a_{\pi}$, both lie in $D$ and that $H$ normalizes $D$. Now $D$ is divisible since $E$ is algebraically closed. Thus there is a divisible hull $X_{\pi}$ of $\left\langle x_{\pi}\right\rangle$ in $D$ such that if $d=\operatorname{diag}\left(\delta_{i}: i \in I\right) \in X_{\pi}$ then $\delta_{i}=\delta_{j}$ whenever $\xi_{i}=\xi_{j}$ and $\delta_{i}=1$ whenever $\xi_{i}=1$. The argument above that $N_{H}(\pi)$ centralizes $x_{\pi}$ clearly yields that $N_{H}(\pi)$ centralizes $X_{\pi}$. Hence $\left\langle X_{\pi}^{H}\right\rangle=\left\langle X_{\pi}^{T}\right\rangle$ and since $\left\langle x_{\pi}^{T}\right\rangle=\times_{T}\left\langle x_{\pi}\right\rangle^{t}$, we have $\left\langle X_{\pi}^{H}\right\rangle=\times_{T} X_{\pi}^{t}$. Thus $\left\langle H, X_{\pi}\right\rangle \cong \mathbb{Q}^{+} \operatorname{wr}_{\Omega} H$.

Suppose $H$ is not transitive on $\Omega$. Let $\Pi$ be a set of representatives for the $H$-orbits of $\Omega$. For each $\pi$ in $\Pi$ repeat the above construction of the representation of $\mathbb{Q}^{+} \mathrm{wr}_{\pi H} H$ in FGL $\left(U_{0}\right)$. Let $\Xi_{\pi}$ denote the set of the non-identity elements $\xi_{i}$ used in this construction. Choose the sets $\Xi_{\pi}$ algebraically independent of each other over $F$. We obtain a faithful representation $\left\langle H, X_{\pi}: \pi \in \Pi\right\rangle \leqslant \mathrm{FGL}\left(U_{0}\right)$ of $\mathbb{Q}^{+} \mathrm{wr}_{\Omega} H$.

The above construction depends upon the set $\Xi=\bigcup_{\pi \in \Pi} \Xi_{\pi}$ of algebraically independent elements of $E$. Suppose that the $\Xi_{j}$ for $j \in J$ are algebraically independent subsets of $E$ over $F$ bijective with $\Xi$ and all algebraically independent of each other over $F$. We then obtain a faithful representation of $\mathbb{Q}^{(J)} \mathrm{wr}_{\Omega} H$ in $\mathrm{FGL}\left(U_{0}\right)$ with the image of the base group diagonal (in the above sense of lying in $D$ ). Here $\mathbb{Q}^{(J)}$ denotes a rational vector space with $J$ as a basis. If $A$ is a torsion-free abelian group, then $A$ embeds into a group of the form $\mathbb{Q}^{(J)}$ for $|J|=\operatorname{rank} A$. Thus $G$ can be embedded into $\operatorname{FGL}\left(U_{0}\right)$ with the image of its base group diagonal and hence completely reducible.

Suppose now that $A \neq\langle 1\rangle$ and $G_{0}$ is completely reducible. Then so too is the normal subgroup $\operatorname{ker} \sigma$ of $G_{0}$ (by the extended Clifford Theorem). Let $W_{1}$ be the sum of the $E H$ composition factors of $E \otimes_{F} V$. Then $W_{1}$ is ker $\sigma$-faithful. Regard $W_{1}$ as an $E G$-module via the obvious map of $G$ onto $H$. Let $W_{2}$ be the sum of the $E G$-composition factors of $U_{0}$ and set $W=W_{1} \oplus W_{2}$. Then $W$ is a completely reducible finitary $E G$-nodule.

Now ker $\sigma$ acts faithfully on $W_{1}$ and $B$ acts faithfully on $W_{2}$. Let $K$ be the kernel of the action of $G$ on $W$. Then $K B$ centralizes $W_{1}$ and hence $K B \cap \operatorname{ker} \sigma=\langle 1\rangle$. The action of $G$ on $W_{2}$ yields that $K \cap B=\langle 1\rangle$, so $K \leqslant C_{G}(B)=B \operatorname{ker} \sigma$, using $A \neq\langle 1\rangle$. Hence

$$
K=K \cap(K B \cap B \operatorname{ker} \sigma)=K \cap B(K B \cap \operatorname{ker} \sigma)=K \cap B=\langle 1\rangle .
$$

Therefore $W$ is a $G$-faithful finitary completely reducible $E G$-module. The proof is complete.

§13. Suppose $G=A \operatorname{wr}_{\Omega} H \leqslant \mathrm{FGL}(V)$, where $A$ is abelian, the base group $B$ is unipotent and the ground field $F$ has characteristic 0 . Let $\left\{\xi_{j}: j \in J\right\}$ be a family of independent indeterminates over $F$, set $E=F\left(\xi_{j}: j \in J\right)$ and let $A^{(J)}$ denote a direct product of $|J|$ copies of $A$ indexed by $J$. Then $A^{(J)} \operatorname{wr}_{\Omega} H$ imbeds into $\operatorname{FGL}\left(E \otimes_{F} V\right)$ with the image of its base group unipotent.

Proof. Since $B \leqslant \mathrm{FGL}(V)$ is abelian and unipotent, $\log$ yields a well-defined isomor- 
phism (multiplication to addition) of $B$ to $\log B \leqslant \operatorname{End}_{F} V$. Then

$$
\sum_{j \in J} \xi_{j} \log B=\oplus_{j \in J} \xi_{j} \log B \leqslant \operatorname{End}_{E}\left(E \otimes_{F} V\right)
$$

is an additive group of commuting nilpotent elements. Hence $X=\exp \left(\sum_{J} \xi_{j} \log B\right)$ is defined and is an abelian unipotent subgroup of $\operatorname{FGL}\left(E \otimes_{F} V\right)$.

Set $B_{j}=\exp \left(\xi_{j} \log B\right)$, so $X=\left\langle B_{j}: j \in J\right\rangle$. If $\Pi_{J} b_{j}=1$, where the $b_{j}$ lie in $B_{j}$ and almost all are 1 , then $\sum \log b_{j}=0$ and each $\log b_{j} \in \xi_{j} \log B$. In view of the direct sum $(*)$, we have that each $\log b_{j}=0$, that each $b_{j}=1$ and that $X$ is the direct product of the $B_{j}$. If $b \in B$ and $h \in H$, a simple calculation yields that $\left(\exp \left(\xi_{j} \log b\right)\right)^{h}=\exp \left(\xi_{j} \log \left(b^{h}\right)\right)$. Thus $B_{j} \cong_{H} B$ and $X$ is $H$-isomorphic to the base group of $A^{(J)} \operatorname{wr}_{\Omega} H$. If $h \in H \cap X$, the entries of $\log h$ lie in both $F$ and $\oplus_{J} \xi_{j} \log B$. Hence $\log h=0$ and $H \cap X=\langle 1\rangle$. Consequently, $\langle H, X\rangle$ is isomorphic to $A^{(J)} \operatorname{wr}_{\Omega} H$. Moreover the base group corresponds, under this isomorphism, to the unipotent group $X$.

$\S 14$. Suppose $G_{0}=\langle a\rangle \operatorname{wr}_{\Omega} H \leqslant \operatorname{FGL}(V)$, where $a$ has infinite order and the base group $B_{0}$ is unipotent. Then for any torsion-free abelian group $A$, the group $G=A \operatorname{wr}_{\Omega} H$ is isomorphic to a finitary linear group of characteristic 0 such that the image of its base group is unipotent.

Proof. Of course here char $F$ must be zero. Now $B_{0}$, being unipotent, stabilizes a $G$ invariant series in $V$ (running from $\{0\}$ to $V$ ). The stability subgroup $S$ of this series in FGL $(V)$ is torsion-free and locally nilpotent. It is also divisible (since $\operatorname{Tr}_{1}(n, F)$ is divisible for every finite $n$ ). Thus $\left\langle a_{\omega}\right\rangle$ has a unique divisible hull $A_{\omega}$ in $S$ and $\left\langle A_{\omega}: \omega \in \Omega\right\rangle$ is the direct product of the $A_{\omega}$. If $h \in H$, then $h$ normalizes $S$, so $\left(A_{\omega}\right)^{h}$ is the divisible hull of $\left\langle a_{\omega}\right\rangle^{h}=\left\langle a_{\omega h}\right\rangle$ and $\left(A_{\omega}\right)^{h}=A_{\omega h}$. Moreover, if $\omega h=\omega$ then $\left(a_{\omega}\right)^{h}=a_{\omega}$ and $h$ centralizes $A_{\omega}$. Therefore $\left\langle H, A_{\omega}: \omega \in \Omega\right\rangle \leqslant \mathrm{FGL}(V)$ is isomorphic to $\mathbb{Q}^{+}{ }^{\mathrm{wr}} \Omega(H$. Note further that its base group is, by construction, unipotent.

By 13 we can now represent faithfully in characteristic zero any group of the form $\mathbb{Q}^{(J)} \operatorname{wr}_{\Omega} H$ with the image of the base group unipotent. But if $A$ is a torsion-free abelian group, then $A$ can be embedded in some group of the form $\mathbb{Q}^{(J)}$ (with $|J|=\operatorname{rank} A$, for example). The lemma follows.

$\S 15$. We have now dealt with both the base-group completely reducible and the basegroup unipotent cases. Unfortunately, one cannot directly separate the general case into these two distinct cases. For example consider the elements

$$
a=\left(\begin{array}{cccc}
x & 0 & 0 & 0 \\
0 & 1 & 0 & 0 \\
0 & 0 & 1 & 0 \\
0 & 0 & 1 & 1
\end{array}\right), \quad h=\left(\begin{array}{cccc}
0 & 1 & 0 & 0 \\
1 & 0 & 0 & 0 \\
0 & 0 & x & 0 \\
0 & 0 & 0 & 1
\end{array}\right)
$$

of $\operatorname{GL}(4, \mathbb{Q}(x))$, where $x$ is an indeterminate over $\mathbb{Q}$. Set $H=\langle h\rangle$ and $G=\langle H, a\rangle$. Then $\mathrm{GL}(4, \mathbb{Q}(x)) \geqslant G \cong C_{\infty}$ wr $C_{\infty}$. 
If $B$ denotes the base group of $G$ in this representation as a wreath product, then $\langle 1\rangle<u(B)<B$. Moreover neither $H . u(B)$ nor $G / u(B)$ have the form of a wreath product $X$ wr $C_{\infty}$ for any group $X$. The trick is to switch to a more convenient representation of $G$. For example $G$ is clearly isomorphic to the subgroup $G_{1}=\left\langle a_{1}, h_{1}\right\rangle$ of $\operatorname{GL}(2, \mathbb{Q}(x))$, where

$$
a_{1}=\left(\begin{array}{ll}
1 & 0 \\
1 & 1
\end{array}\right), \quad h_{1}=\left(\begin{array}{ll}
x & 0 \\
1 & 1
\end{array}\right)
$$

and the base group $B_{1}$ of $G_{I}$ corresponding to $B$ is unipotent. Then we are in the situation of 14 .

$\S 16$. Suppose $G=\langle a\rangle \operatorname{wr}_{\Omega} H \leqslant \operatorname{FGL}(V)$, where $|a|=\infty$, char $F=0$ and $F$ is algebraically closed. Then $G$ can be embedded into $\operatorname{FGL}\left(V^{(3)}\right)$ such that the image of the base group $B$ of $G$ is unipotent.

Proof. The additive and multiplicative groups of $F$ are as follows:

$$
(F,+) \cong \mathbb{Q}^{(|F|)}, \quad \text { i.e. } \operatorname{dim}_{\mathbb{Q}} F=|F|, \text { and } F^{*} \cong(\mathbb{Q} / \mathbb{Z}) \oplus \mathbb{Q}^{(|F|)}
$$

Thus there is a group homomorphism $\varepsilon$ of the additive group of $F$ onto the multiplicative group $F^{*}$ of $F$.

The abelian group $B$ has a Jordan decomposition $B \leqslant B_{u} \times B_{d} \leqslant$ FGL $(V)$, say $b=b_{u} b_{d}$ for $b \in B$ (see $\left.[3,2.3]\right)$. Consider $W=\left[V, B_{d}\right]=\sum_{b \in B} V\left(b_{d}-1\right)$. If $b \in B_{d}$, then $[V, b]$ is a finite-dimensional $F B_{d^{-}}$-module and as such is a sum of one-dimensional $F B_{d^{-}}$ modules. Thus $W$ is a direct sum of one-dimensional $F B_{d^{-}}$modules. Let $W=\oplus_{i \in I} W_{i}$, where the $W_{i}$ are the non-zero $F B_{d}$-homogeneous components of $W$. Since $H$ normalizes $B$, so $H$ normalizes $B_{d}$, the space $W$ is an $H$-submodule of $V$ and $H$ permutes the set $\left\{W_{i}: i \in I\right\}$. Transfer this action to $I$; i.e. set $W_{i h}=W_{i} h$ for all $i$ in $I$ and $h$ in $H$.

Let $\Pi$ be a set of representatives for the $H$-orbits of $\Omega$ and let $\pi \in \Pi$. Now $B_{d}$ acts on each $W_{i}$ as a group of scalars. Let $a_{\pi, d}$ (the diagonalizable component of $a_{\pi}$ ) act on $W_{i}$ as the scalar $\eta_{i, \pi}$. Then for $h$ in $H$ we have that $a_{\pi h, d}=\left(a_{\pi, d}\right)^{h}$ acts on $W_{i h}$ as $\eta_{i, \pi}$ and on $W_{i}$ as $\eta_{i h^{-1}, \pi}$. Let $Y=\left\{\eta_{i, \pi}: i \in I \& \pi \in \Pi\right\}$. For each $\eta \in Y$ pick $\xi$ in $F$ with $\xi \varepsilon=\eta$ and $\xi=0$ if $\eta=1$. Then we have a subset $X=\left\{\xi_{i, \pi}: i \in I \& \pi \in \Pi\right\}$ of $F$ such that $\xi_{i, \pi} \varepsilon=\eta_{i, \pi}, \xi_{i, \pi}=0$ if $\eta_{i, \pi}=1$ and $\xi_{i, \pi}=\xi_{j, \kappa}$ if $\eta_{i, \pi}=\eta_{j, \kappa}$; this for all allowed choices of the suffices. In particular $\varepsilon$ restricts to a homomorphism of the additive group $\langle X\rangle$ onto the multiplicative group $\langle Y\rangle$.

Let $x_{\pi}$ denote the 'diagonal' element of $\operatorname{End}_{F} W$, where $x_{\pi}$ acts as the scalar $\xi_{i, \pi}$ on $W_{i}$ for all $i$. Then $\left(x_{\pi}\right)^{h}$ acts on $W_{i}$ as $\xi_{i h^{-1}, \pi}$. Suppose $\pi h=\pi k$ for some $h, k \in H$. Then $a_{\pi h, d}=a_{\pi k, d}$ acts on $W_{i}$ as both $\eta_{i h^{-1}, \pi}$ and $\eta_{i k^{-1}, \pi}$. Hence these are equal, so $\xi_{i h^{-1}, \pi}=\xi_{i k^{-1}, \pi}$ for each $i$. Therefore $\left(x_{\pi}\right)^{h}=\left(x_{\pi}\right)^{k}$. Set $x_{\pi h}=\left(x_{\pi}\right)^{h}$ for all $h \in H$; by the above, this is well-defined.

Define $\lambda: B \rightarrow \operatorname{End}_{F} W$ by $a_{\omega} \mapsto x_{\omega}$ for each $\omega$ in $\Omega$. Since $B$ is free abelian on the $a_{\omega}$, so $\lambda$ is a well-defined group homomorphism. The homomorphism $\varepsilon:\langle X\rangle \rightarrow\langle Y\rangle$ above defines a group homomorphism $\mu$ (addition to multiplication) of $\left\langle x_{\omega}: \omega \in \Omega\right\rangle$ to $\left.B_{d}\right|_{W}$ 
such that $\mu:\left.x_{\omega} \mapsto a_{\omega, d}\right|_{W}$. Then $\lambda \mu$ is the natural homomorphism of $B$ onto $\left.B_{d}\right|_{W}$ given by $\left.b \mapsto b_{d}\right|_{W}$ for all $b$ in $B$. Clearly $\lambda$ and $\mu$ are $H$-maps. Suppose $b \in B$ with $b \lambda=0$. Then $\left.b_{d}\right|_{W}=b \lambda \mu=1$. But $b_{d}$ is diagonalizable on $V$ and now is the identity on both $W$ and $V / W$, so $b_{d}=1$. If also $b_{u}=1$, then $b=b_{u} b_{d}=1$.

We define a homomorphism $\phi$ of $G$ into $\operatorname{FGL}\left(V \oplus W^{(2)}\right)$ as follows. For $h$ in $H$ and $b$ in $B$, set

$$
h \phi=\left(\begin{array}{ccc}
h & 0 & 0 \\
0 & \left.h\right|_{W} & 0 \\
0 & 0 & \left.h\right|_{W}
\end{array}\right) \quad \text { and } \quad b \phi=\left(\begin{array}{ccc}
b_{u} & 0 & 0 \\
0 & 1 & b \lambda \\
0 & 0 & 1
\end{array}\right) .
$$

Since $b \lambda$ has only a finite number of non-zero entries (each $\operatorname{dim}_{F} W_{i}$ is finite by finitariness and almost all the $\eta_{i, \pi}$ for a given $\pi$ in $I$ are 1 ), so $b \phi$ is finitary. Also $\left(x_{\omega}\right)^{h}=x_{\omega h}$ and $\left(b_{u}\right)^{h}=\left(b^{h}\right)_{u}$, so the above defines a homomorphism $\phi$ of $G$ into $\operatorname{FGL}\left(V \oplus W^{(2)}\right)$. Clearly, $\phi$ is one-to-one on $H$ and the previous paragraph yields that $\phi$ is one-to-one on $B$. If $h \in H$ and $b \in B$ with $h \phi=b \phi$, then $b \lambda=0$, so $b_{d}=1$; see the previous paragraph. Hence $b=b_{u}=h \in H \cap B=\langle 1\rangle$. Therefore $H \phi \cap B \phi=\langle 1\rangle$. Consequently $\phi$ is an embedding of $G$. Clearly, the image $B \phi$ of $B$ is unipotent. Finally the containment of $W$ in $V$ yields an embedding of $\operatorname{FGL}\left(V \oplus W^{(2)}\right)$ into $\operatorname{FGL}\left(V^{(3)}\right)$ mapping unipotent elements to unipotent elements. The proof is complete.

$\S 17$. Proof of the proposition. Clearly, we may assume $A_{0}=\langle a\rangle$ is infinite cyclic. Assume $G_{0} \leqslant \mathrm{FGL}(V)$, where char $F=p$. We may extend the ground field, so let $F$ be algebraically closed. Suppose $p=0$. By 16 we may also assume that the base group $B_{0}$ of $G_{0}$ is unipotent. The required conclusion then follows from 14 .

Now assume $p>0$. Here $B_{0}$ is unipotent-free. Let $W$ be the direct sum of $V$ and the direct sum of the $F G_{0}$-composition factors of $V$, where $G_{0}$ acts on the summand $V$ via $G_{0} \rightarrow H \leqslant \mathrm{FGL}(V)$. By (the extended) Clifford Theorem, $W$ is a $G_{0}$-faithful, $F B_{0}$-completely reducible, finitary $F G_{0}$-module. The proposition now follows from 12 .

$\S 17$. Remark. In the proof of 16 we have the minor complication of replacing the given action on $V$ by the one on $W$. The reason for this is that although $W$ is always completely reducible as $F B_{d}$-module, strangely $V$ might not be. For consider the example $B \leqslant G \leqslant$ FGL $(V)$ on pp. 173-174 of [5]. Choose $D$ there to be an algebraically closed field $F$. Then $B_{d}=B$. Also, in the notation of $[5]$, we have $[V, B] \leqslant W<V$, where $W$ is a direct sum of one-dimensional $F B$-submodules and $\operatorname{dim}_{F}(V / W)=1$. It is easy to check from the definitions in [5] that $C_{W}(B)=\{0\}$. If $V$ is completely reducible as $F B$-module, then

$$
V=C_{V}(B) \oplus W .
$$

But $C_{V}(B)$ and $W$ are $F G$-submodules of $V$ with $W$ irreducible and $\operatorname{dim}_{F} C_{V}(B)=1$, and yet $V$ is not completely reducible as $F G$-module. This contradiction proves that $V$ is not completely reducible as $F B_{d}$-module. 


\section{References}

1. R. E. Phillips, Finitary linear groups; a survey, in Finite and locally finite groups, pp. 111146, NATO ASI Series C 471 (Kluwer, Dordrecht, 1995).

2. B. A. F. Wehrfritz, Locally soluble finitary skew linear groups, J. Algebra 160 (1993), 226-241.

3. B. A. F. Wehrfritz, Nilpotence in finitary linear groups, Michigan Math. J. 40 (1993), 419-432.

4. B. A. F. Wehrfritz, Irreducible locally nilpotent finitary skew linear groups, Proc. Edinb. Math. Soc. 38 (1995), 63-76.

5. B. A. F. Wehrfritz, The complete reducibility of locally completely reducible finitary linear groups, Bull. Lond. Math. Soc. 29 (1997), 173-176.

6. B. A. F. Wehrfritz, The linearity of wreath products, Mathematika 44 (1997), 357-367.

7. B. A. F. Wehrfritz, On the finitary linearity of wreath products, Algebra Colloq. 6 (1999), 23-32. 